\title{
ALERT-LDL: adherence to guidelines in the treatment of patients with dyslipidemia
}

\author{
Giorgio Bosso ${ }^{1}$ Mariarosaria De Luca ${ }^{2}$. Giovanni Alma ${ }^{1}$. Vincenzo Carbone ${ }^{1} \cdot$ Ferdinando Ferrara ${ }^{1}$. \\ Biagio Fimiani ${ }^{1} \cdot$ Franco Guarnaccia $^{1}$ - Alessandro landolo ${ }^{1}$. Sabato Murolo ${ }^{1} \cdot$ Maurizio Olivares $^{1}$. \\ Emanuele Romeo ${ }^{1}$. Giosuè Santoro ${ }^{1}$. Antonio Valvano ${ }^{1}$. Giovanni Zito ${ }^{1} \cdot$ Ugo Oliviero $^{1,2}$
}

Received: 13 April 2021 / Accepted: 14 July 2021 / Published online: 24 July 2021

(c) The Author(s) 2021

\begin{abstract}
The association between LDL-c levels and cardiovascular outcomes suggests tailoring lipid-lowering therapies according to total cardiovascular risk. We aimed to evaluate the adherence to guidelines-oriented dyslipidaemia's treatment in an outpatient population referring to ARCA cardiologists, and assess the efficacy of treatment's optimization for each specific level of risk. Three thousand seventy-five patients enrolled in this prospective study were classified according to cardiovascular risk category, and their therapies were optimized. At the beginning and the 3 month follow-up visit, LDL-c data were collected, and further therapies were prescribed to the patients that did not reach the target. A significant LDL-c reduction was observed in all subgroups at different cardiovascular risk at the end of the study $(p<0.05)$. The number of patients assuming statins, both in monotherapy and in combination with ezetimibe, increased during the follow-up $(63 \%$ at the enrollment vs $89 \%$ after 12 months). At the enrollment, only $1.4 \%$ of patients were treated with PCSK-9 inhibitors while after 12 months the percentage increased both in high (5.8\%) and very high-risk (18.4\%) patients. At the beginning of the study, only 698/3075 patients (22.7\%) reached lipid targets. At the end of the study, carried out by the referring cardiologists in the pertaining healthcare districts and specifically aimed to control the lipid profile, the percentage of patients on target increased in all risk categories (68.5\%). Our results suggest carefully implementing measures that encourage outpatients and their cardiologists to achieve the targeted lipid profile according to cardiovascular risk.
\end{abstract}

Keywords Dyslipidaemia $\cdot$ Cholesterol $\cdot$ Guidelines $\cdot$ Adherence $\cdot$ Targets $\cdot$ Statins

\section{Introduction}

Dyslipidaemia represents a relevant and preventable component of cardiovascular burden and the serum levels of cholesterol are considered one of the most useful parameters to define cardiovascular risk in adults [1-3]. In particular, several trials have demonstrated a strong relationship between cholesterol levels and the incidence of coronary artery disease [4], as well as between the time of exposure to high values of LDL cholesterol (LDL-c) and cardiovascular risk $[5,6]$.

Ugo Oliviero

ugo.oliviero@unina.it

1 ARCA (Associazioni Regionali Cardiologi Ambulatoriali), Campania, Italy

2 Department of Translational Medical Sciences, University Federico II, Via Pansini, 5, 80131 Naples, Italy
Due to the evidence on the effectiveness of lowering lipid levels in improving cardiovascular outcomes [7-10], a relevant interest is growing in lowering cholesterol levels and identifying individuals who could benefit from cholesterollowering interventions. Among them, very high-risk patients should achieve an absolute LDL-c goal of $<55 \mathrm{mg} / \mathrm{dl}$, while high-risk patients should aim for a target of $<70 \mathrm{mg} / \mathrm{dl}$. Guidelines did not identify specific goals for HDL-C or triglycerides levels but suggest clinical judgment and particular consideration to these parameters when hypolipidemic treatments are instituted [1].

Despite the well-known dyslipidaemia's cardiovascular effects and the large prescription of antidyslipidaemic drugs, there are some concerns regarding the rigorous adherence to the guidelines' suggested lipid targets in real-world practice $[11,12]$.

ALERT-LDL was a prospective study designed to evaluate the adherence to guidelines-oriented dyslipidaemia's 
treatment in an outpatient population referring to ARCA (Associazioni Regionali Cardiologi Ambulatoriali) cardiologists, and to assess the efficacy of the treatment's optimization for each specific level of risk.

\section{Materials and methods}

\section{Study protocol}

Among the 3648 outpatients consecutively referred to 12 ARCA cardiologists of the pertaining healthcare districts from March 2019 to May 2019, 3075 patients (1602 female/1473 male) were included and completed the study. Thirteen patients, firstly scheduled, died during the observation period, did not complete the program, and were excluded from the analysis.

The inclusion criteria were age between 45 and 75 years and diagnosis of dyslipidemia, defined according to European Society of Cardiology (ESC) guidelines [1], while the exclusion criteria were the current involvement in other clinical trials, malignant neoplasms that could reduce life expectation, and the presence of advanced chronic kidney disease \{estimated Glomerular Filtration Rate $[e G F R] \leq 15 \mathrm{ml} / \mathrm{min}$ per $1.73 \mathrm{~m}^{2}$, according to the Chronic Kidney Disease Epidemiology Collaboration (CKD-EPI) equation .

All the enrolled patients signed a written informed consent and agreed to participate in a 12 month follow-up program, and the principles outlined in the Declaration of Helsinki were followed.

During the first visit, each patient underwent a detailed clinical evaluation, including medical history recording, concomitant treatments, physical examination, and assessment of anthropometric parameters and vital signs.

Data on lipid blood levels (total cholesterol, LDL cholesterol, HDL cholesterol, triglyceridemia) within the last 2 weeks were collected and compared to the targets suggested by ESC guidelines. The therapy was optimized (increasing dose and/or adding drug) in patients who did not reach the target according to the cardiovascular risk profile.

During the 3 month follow-up visit (intermediate visit) and the 12 month follow-up visit (final visit), LDL-cholesterol data were collected again, to verify if therapy optimization had allowed the achievement of suggested targets, and further therapies were prescribed to patients that had not yet reached the LDL-cholesterol targets according to ESC guidelines [1]. Side effects and compliance to drug assumption were also recorded at the intermediate and final visits.

Coming data by every ARCA cardiologist pertaining healthcare district were stored in anonymized patients' schedules and sent to the coordinating center for statistical analysis.

\section{ALERT-LDL program}

ARCA cardiologists were advised to closely adhere to the ALERT-LDL program, which consisted of:

- Scheduled 3 month and 12 months follow-up visits for all patients enrolled.

- Accurate reassessment of cardiovascular risk and lipidic levels at each visit.

- The well-defined and shared objective of reducing hypercholesterolaemia until to achieve guidelines suggested targets.

- Patient education sessions on the need to undergo a hypolipidemic diet and perform physical activity programs. Physicians encouraged to consume fiber, fish [1,2], unsalted nuts [13], fruits [2,3], and vegetables (2-3 servings per day), and to restrict the assumption of saturated fatty acids, sweets, and alcoholic beverages. Overweight and obese people were referred for a dietary consultation with a nutrition specialist. Moreover, subjects were advised to perform at least 150 min a week of physical exercise with appropriate types of activities and intensity. They were helped to set personal goals to achieve the benefits.

- Promotion of all measures that can increase patient compliance (focusing on patient knowledge of therapy's benefits and risks, prescribing single tablet combination, checking compliance at each visit, sharing decisions).

- Availability of an e-mail service for patients who needed further information about medical treatment and lifestyle interventions during the follow-up.

\section{Cardiovascular risk stratification}

Since new guidelines were proposed after the beginning of the study [1], the placement of each patient within a certain risk class was verified and remained unchanged from the previous evaluation [14].

The total cardiovascular risk, defined as the likelihood of a person developing a fatal cardiovascular event over the next 10 years, was calculated according to both the SCORE (Systematic Coronary Risk Estimation) system and the evaluation of clinical history. Patients with overt cardiovascular disease (i.e. chronic coronary syndrome, previous stroke, and peripheral arterial disease), diabetics with target organ damage, or patients with at least three major risk factors, (severe chronic kidney disease, familial hypercholesterolemia, or a calculated SCORE > 10\%) were considered at very high-risk. Patients with markedly elevated single risk factors (in particular triglycerides $>310 \mathrm{mg} / \mathrm{dL}$, LDL cholesterol $>190 \mathrm{mg} / \mathrm{dl}$, or blood pressure $\geq 180 / 110 \mathrm{mmHg}$ ), patients with diabetes for 
more than 10 years or together with an additional risk factor, with moderate CKD or with a calculated SCORE $>5 \%$ and $<10 \%$ were considered at high risk. Diabetic patients with a disease duration less than 10 years and without other risk factors or people with a calculated SCORE between 1 and 5\% were considered at moderate cardiovascular risk.

Finally, the low-risk category included all subjects with a calculated SCORE $<1 \%$ and no previous cardiovascular disease.

\section{Lipids' therapeutic targets}

ARCA cardiologists set cholesterol targets based on the latest guidelines [1]. In particular, the new guidelines suggest that an LDL reduction $\geq$ of $50 \%$ from baseline with a target $<55 \mathrm{mg} / \mathrm{dl}$ has to be achieved by the individuals at very high risk; an LDL target $<70 \mathrm{mg} / \mathrm{dl}$ can be acceptable in high-risk patients and LDL values $<100 \mathrm{mg} / \mathrm{dl}$ and $<116 \mathrm{mg} / \mathrm{dl}$ are recommended for moderate-risk and low-risk subjects, respectively.

\section{Statistical analysis}

Categorical variables were expressed as absolute number and percentage. Continuous variables were expressed as mean \pm standard deviation. The Shapiro-Wilk test was used to determine if the continuous variables were normally distributed or not. To assess statistical differences for cholesterol levels across the follow-up visit (initial vs intermediate vs final visit) ANOVA test for normally distributed variables or Friedman test for not normally distributes variables were performed. A $p$ value less than 0.05 was considered statistically significant. Statistical analysis was performed using the SPSS package, version 22 (SPSS Inc., Chicago, IL).

\section{Results}

Among the 3075 patients enrolled, 298 (9.7\%) subjects were considered at low risk, $340(11.1 \%)$ at moderate risk, 550 (17.9\%) at high risk, and $1887(61.3 \%)$ at very high risk.

The anthropometric and clinical characteristics of the study population according to cardiovascular risk stratification at baseline are summarized in Table 1. The most prevalent comorbidity was hypertension, both in the total study population (2231 patients, $72.5 \%$ ) and in all the subgroups at different cardiovascular risk, followed by diabetes and chronic coronary syndrome $(46.6 \%$ and $35.5 \%$ of the total population, respectively).

Table 2 shows total cholesterol, LDL and HDL cholesterol and triglyceridemia at the enrollment and after 3 and 12 months follow-up according to the different cardiovascular risk categories. After 3 months total cholesterol and LDL-c resulted reduced in all the subgroups at different cardiovascular risk $(p<0.05)$. Further intensification of therapy in patients who had not yet reached their lipid targets led to an additional reduction in LDL-c values after 12 months compared with the baseline $(p<0.05)$ and the 3 month levels $(p=n s)$. Accordingly, triglycerides resulted reduced and HDL cholesterol increased in all risk categories patients at the intermediate and final visit in respect to baseline $(p<0.05)$.

Table 3 and Fig. 1 report hypolipidemic therapies at the enrollment and after 3 and 12 months follow-up for the different risk categories. Patients assuming statins, both in monotherapy and in combination with ezetimibe, increased during the follow-up (63\% at the enrollment vs $89 \% 1$ year later, $p<0.05$ ). At the beginning of the study $55.0 \%$ of them were treated with high-intensity statins (atorvastatin 36.9\% and rosuvastatin $18.1 \%$ ) and $45.0 \%$ with moderate-intensity statins (simvastatin $40.3 \%$, pravastatin $3.7 \%$, lovastatin $1.0 \%)$. At the end of the study, the use of high-intensity
Table 1 Anthropometric and clinical characteristics of the study population

\begin{tabular}{llllll}
\hline & $\begin{array}{l}\text { Low } \\
(n=298)\end{array}$ & $\begin{array}{l}\text { Moderate } \\
(n=340)\end{array}$ & $\begin{array}{l}\text { High } \\
(n=550)\end{array}$ & $\begin{array}{l}\text { Very high } \\
(n=1887)\end{array}$ & $\begin{array}{l}\text { Total } \\
(n=3075)\end{array}$ \\
\hline Sex Female, $n(\%)$ & $138(46.3)$ & $171(50.3)$ & $296(53.8)$ & $997(52.8)$ & $1602(52.1)$ \\
Age, years & $64.7 \pm 8.3$ & $64.9 \pm 8.5$ & $65.2 \pm 8.5$ & $64.8 \pm 8.3$ & $64.7 \pm 8.3$ \\
BMI, kg/m ${ }^{2}$ & $28.2 \pm 4.7$ & $28.3 \pm 4.7$ & $28.6 \pm 4.9$ & $28.2 \pm 4.8$ & $28.2 \pm 4.8$ \\
Hypertension, $n(\%)$ & $110(36.9)$ & $189(55.6)$ & $399(72.5)$ & $1533(81.2)$ & $2231(72.5)$ \\
Diabetes, $n(\%)$ & - & $26(7.6)$ & $336(61.1)$ & $1071(56.7)$ & $1433(46.6)$ \\
Chronic coronary syn- & - & - & - & $1092(57.9)$ & $1092(35.5)$ \\
$\quad$ drome, $n(\%)$ & & - & - & $221(11.7)$ & $221(7.2)$ \\
Severe CKD, $n(\%)$ & - & $65(19.1)$ & $101(18.4)$ & $336(17.8)$ & $547(17.8)$ \\
Smokers, $n(\%)$ & $45(15.1)$ & & & &
\end{tabular}

Data expressed as mean \pm standard deviation or frequencies or percentage when indicated $B M I$ body mass index, $S B P$ systolic blood pressure, $D B P$ diastolic blood pressure, $C K D$ chronic kidney disease 
Table 2 Lipid profile at baseline, intermediate (after 3 months) and final visit (after 12 months)

\begin{tabular}{|c|c|c|c|c|c|c|c|c|}
\hline & $\begin{array}{l}\text { Low } \\
(n=298)\end{array}$ & $\begin{array}{l}\text { Moderate } \\
(n=340)\end{array}$ & $\begin{array}{l}\text { High } \\
(n=550)\end{array}$ & $\begin{array}{l}\text { Very high } \\
(n=1887)\end{array}$ & $\begin{array}{l}\text { Total } \\
(n=3075)\end{array}$ & $p^{*}$ & $p^{* *}$ & $p^{* * *}$ \\
\hline \multicolumn{9}{|l|}{ Tot-cholesterol } \\
\hline - Baseline & $191.2 \pm 40.5$ & $193.7 \pm 39.6$ & $204.3 \pm 41.4$ & $206.2 \pm 40.3$ & $199.5 \pm 40.3$ & $<0.05$ & $<0.05$ & ns \\
\hline - Intermediate visit & $168.3 \pm 31.0$ & $171.6 \pm 30.7$ & $152.9 \pm 31.1$ & $154.7 \pm 30.6$ & $158.5 \pm 30.7$ & & & \\
\hline - Final visit & $145.1 \pm 35.6$ & $149.5 \pm 33.3$ & $147.3 \pm 30.8$ & $142.6 \pm 32.9$ & $144 \pm 47.8$ & & & \\
\hline \multicolumn{9}{|l|}{ LDL cholesterol } \\
\hline - Baseline & $122.7 \pm 35.3$ & $126.5 \pm 35.5$ & $131.8 \pm 37.1$ & $141.5 \pm 35.3$ & $135 \pm 37.0$ & $<0.05$ & $<0.05$ & ns \\
\hline - Intermediate visit & $95.5 \pm 41.8$ & $98.1 \pm 41.9$ & $80.7 \pm 26.6$ & $81.9 \pm 26.3$ & $85 \pm 39.2$ & & & \\
\hline - Final visit & $75.5 \pm 26.5$ & $78.3 \pm 32.6$ & $73.3 \pm 24.6$ & $63.4 \pm 18.9$ & $67.6 \pm 26.3$ & & & \\
\hline \multicolumn{9}{|l|}{ HDL cholesterol } \\
\hline - Baseline & $47.5 \pm 12.4$ & $48.4 \pm 12.2$ & $49.5 \pm 12.4$ & $45.7 \pm 12.2$ & $47.2 \pm 12.2$ & $<0.05$ & $<0.05$ & ns \\
\hline - Intermediate visit & $51.6 \pm 12.2$ & $49.5 \pm 12.5$ & $51.5 \pm 12.9$ & $47.4 \pm 12.6$ & $49.5 \pm 12.6$ & & & \\
\hline - Final visit & $53.1 \pm 11.8$ & $49.9 \pm 12.0$ & $52.2 \pm 11.5$ & $48.8 \pm 13.0$ & $50.1 \pm 12.0$ & & & \\
\hline \multicolumn{9}{|l|}{ Triglycerides } \\
\hline - Baseline & $145.2 \pm 65.1$ & $147.3 \pm 62.6$ & $150.4 \pm 66.7$ & $152.0 \pm 69.8$ & $148.5 \pm 69.8$ & $<0.05$ & $<0.05$ & ns \\
\hline - Intermediate visit & $118.2 \pm 41.2$ & $121.5 \pm 41.9$ & $120.1 \pm 43.7$ & $122.9 \pm 41.3$ & $120.2 \pm 41.7$ & & & \\
\hline - Final visit & $106 \pm 33.1$ & $114.3 \pm 40.2$ & $112.5 \pm 35.6$ & $106.8 \pm 40.1$ & $110.7 \pm 38.9$ & & & \\
\hline
\end{tabular}

Data expressed as mean \pm standard deviation

$p^{*}$ Comparison between baseline and intermediate data in the study population and in subgroups at different cardiovascular risk

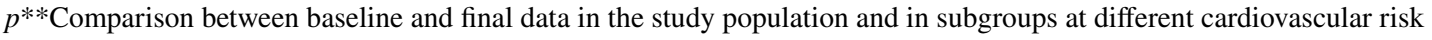

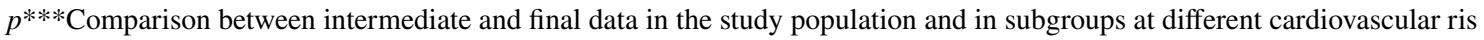

statins increased from 55 to $71.2 \%$, and moderate-intensity statins treatment reduced from 45 to $28.8 \%$. The percentage of patients assuming Nutraceuticals, Fibrates and Omega 3 remained essentially unchanged during the follow-up. At the enrollment, only 42 patients at very high risk $(2.2 \%$ of the total very high-risk population) were treated with PCSK-9 inhibitors, whereas, at the end of the study the percentage increased both in high $(5.8 \%)$ and very high-risk patients $(18.4 \%, p<0.05)$.

Figure 2 shows the percentage of patients that reached the lipid target at the beginning of the study and after 3 and 12 months follow-up. At the beginning, only 698 patients $(22.7 \%)$ showed cholesterol levels comprised in the guidelines' suggested referring values. The percentage was even lower in very high-risk patients (170 patients, $9.0 \%$ of the 1887 patients at very high risk). In this subgroup, the number of patients who reached the target increased after 3 (41.6\%, $p<0.05$ versus patients on target at baseline) and 12 months $(55.8 \%, p<0.01$ vs baseline) follow-up. This trend was observed in all risk categories resulting in a significant increase in the percentage of patients on target after 3 months $(52.9 \%)$ and at the end of the study $(68.5 \%$ of the study population, $p<0.01$ vs patients on target at the enrollment).

Major cardiovascular events (non-fatal myocardial infarction, stroke, other ischemic events) occurred in 29 patients at high and very high risk $(0,94 \%$ of the total population)
(Table 4). Among these, five high-risk patients at the enrollment experienced a major cardiovascular event during the follow-up [2, 3] and were therefore considered at very high risk. Nine low-risk patients at the beginning developed diabetes and were classified as moderate-risk subjects. All these patients did not reach the lipid targets suggested for their risk category either at the beginning of the study or at the follow-up visits.

Multivariate analysis among patient's characteristics (sex, age, BMI) and comorbidities (hypertension, CKD, diabetes, chronic coronary syndrome) and the achievement of lipid target for each $\mathrm{CV}$ risk category were performed. None of the variables resulted associated with the achievement of lipid target for each CV group.

\section{Discussion}

Dyslipidaemia is one of the main determinants of cardiovascular burden and guidelines recommend very stringent LDL-c targets particularly in high-risk patients [1]. In reallife clinical practice the achievement of these therapeutic objectives may be hampered by different causes such as therapeutic inertia, long waiting time between follow-up visits, poor patient compliance, low lipid measurement rates, occurrence of adverse events [15]. 
Table 3 Hypolipidemic drugs prescribed at the baseline, intermediate (after 3 months) and final visit (after 12 months)

\begin{tabular}{|c|c|c|c|c|c|}
\hline & $\begin{array}{l}\text { Low } \\
(n=298)\end{array}$ & $\begin{array}{l}\text { Moderate } \\
(n=340)\end{array}$ & $\begin{array}{l}\text { High } \\
(n=550)\end{array}$ & $\begin{array}{l}\text { Very high } \\
(n=1887)\end{array}$ & $\begin{array}{l}\text { Total } \\
(n=3075)\end{array}$ \\
\hline \multicolumn{6}{|c|}{ Statin monotherapy, $n(\%)$} \\
\hline - Baseline & $116(38.9)$ & $178(52.4)$ & $217(39.5)$ & $1060(56.1)$ & $1571(51.1)$ \\
\hline - Intermediate visit & $203(68.1)$ & $213(62.7)$ & $341(62.0)$ & $614(32.6)$ & $1371(44.6)$ \\
\hline - Final visit & $215(72.1)$ & $255(75.0)$ & $401(72.9)$ & $528(27.9)$ & $1399(45.5)$ \\
\hline \multicolumn{6}{|c|}{ Ezetimibe monotherapy, $n(\%)$} \\
\hline - Baseline & - & $37(10.9)$ & $28(5.1)$ & $105(5.7)$ & $170(5.5)$ \\
\hline - Intermediate visit & $15(5.0)$ & $41(12.1)$ & $42(7.6)$ & $84(4.6)$ & $182(5.9)$ \\
\hline - Final visit & $18(6.0)$ & $40(11.8)$ & $47(8.5)$ & $81(4.3)$ & $186(6.0)$ \\
\hline \multicolumn{6}{|c|}{ Statin + ezetimibe, $n(\%)$} \\
\hline - Baseline & $39(13.0)$ & $5(1.5)$ & $21(3.8)$ & $297(15.7)$ & $362(11.8)$ \\
\hline - Intermediate visit & $42(14.0)$ & $21(6.2)$ & $69(12.5)$ & $1025(54.3)$ & $1157(37.6)$ \\
\hline - Final visit & $63(21.1)$ & $38(11.2)$ & $91(16.5)$ & $1151(61.0)$ & $1343(43.7)$ \\
\hline \multicolumn{6}{|l|}{ Nutraceuticals, $n(\%)$} \\
\hline - Baseline & $8(2.7)$ & $46(13.5)$ & $35(6.4)$ & $64(3.4)$ & $153(5.0)$ \\
\hline - Intermediate visit & $10(3.4)$ & $64(18.8)$ & $38(6.9)$ & $42(2.2)$ & $154(5.0)$ \\
\hline - Final visit & $10(3.4)$ & $60(17.7)$ & $38(6.9)$ & $33(1.75)$ & $141(4.6)$ \\
\hline \multicolumn{6}{|l|}{ Fibrates, $n(\%)$} \\
\hline - Baseline & $31(10.4)$ & $11(3.2)$ & $23(4.2)$ & $148(7.8)$ & $213(6.9)$ \\
\hline - Intermediate visit & $16(5.4)$ & $8(2.4)$ & $25(4.5)$ & $140(7.4)$ & $189(6.1)$ \\
\hline - Final visit & $15(5.0)$ & $8(2.4)$ & $28(5.1)$ & $131(6.9)$ & $182(5.9)$ \\
\hline \multicolumn{6}{|l|}{ Omega $3, n(\%)$} \\
\hline - Baseline & $13(4.4)$ & $21(6.2)$ & $18(3.3)$ & $61(3.2)$ & $113(3.7)$ \\
\hline - Intermediate visit & $53(17.8)$ & $52(15.3)$ & $31(5.6)$ & $96(5.1)$ & $232(7.5)$ \\
\hline - Final visit & $58(19.4)$ & $55(16.2)$ & $33(6.0)$ & $94(4.9)$ & $240(7.8)$ \\
\hline \multicolumn{6}{|c|}{ PCSK-9 inhibitors, $n(\%)$} \\
\hline - Baseline & - & - & - & $42(2.2)$ & $42(1.4)$ \\
\hline - Intermediate visit & - & - & $21(3.8)$ & 169 (8.9) & $190(5.5)$ \\
\hline - Final visit & - & - & $32(5.8)$ & $348(18.4)$ & $380(12.4)$ \\
\hline
\end{tabular}

Fig. 1 Hypolipidemic drugs in the study population at the baseline, intermediate (after 3 months) and final (after 12 months) visit

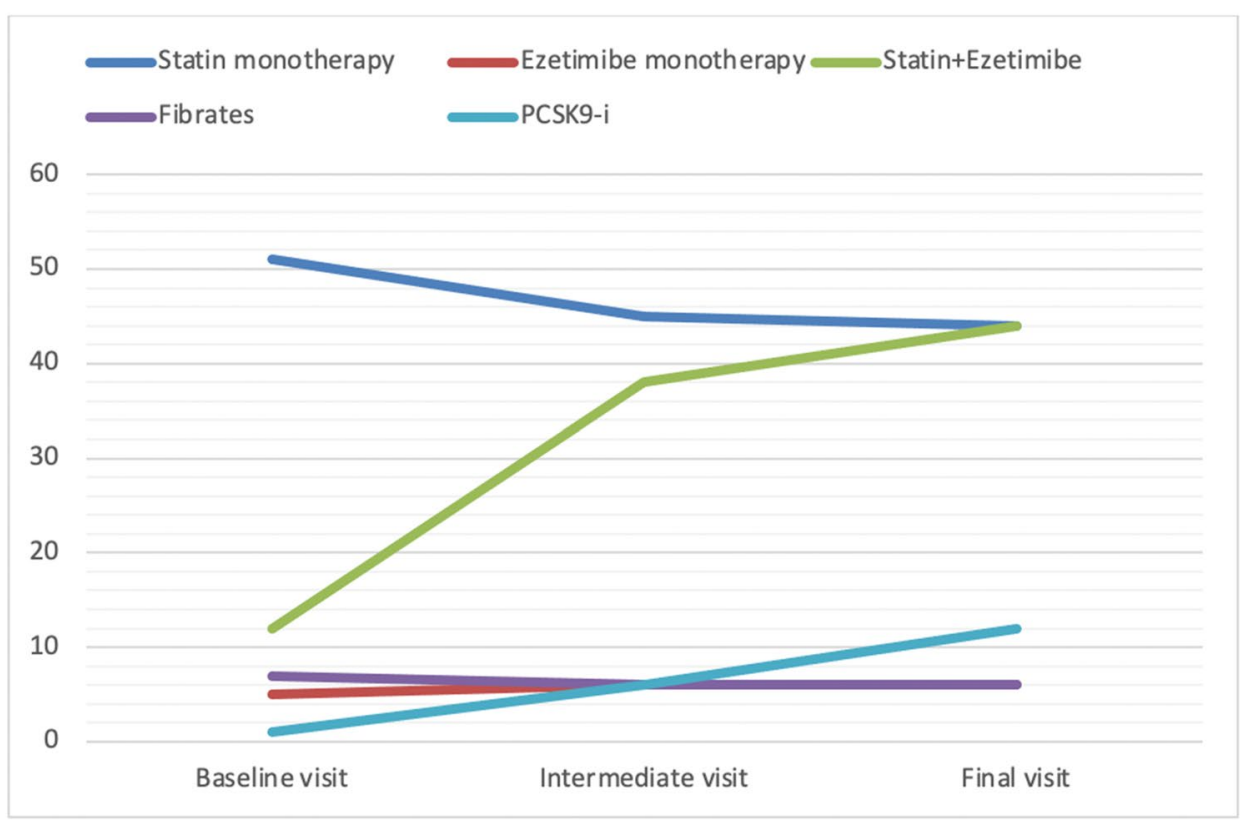


Fig. 2 Patients at different cardiovascular risk on target at the baseline, intermediate (after 3 months) and final visit (after 12 months).*Comparison between intermediate visit and baseline in the study population and in subgroups at different cardiovascular risk, $p<0,05 . * *$ Comparison between final visit and baseline in the study population and in subgroups at different cardiovascular risk, $p<0,01$

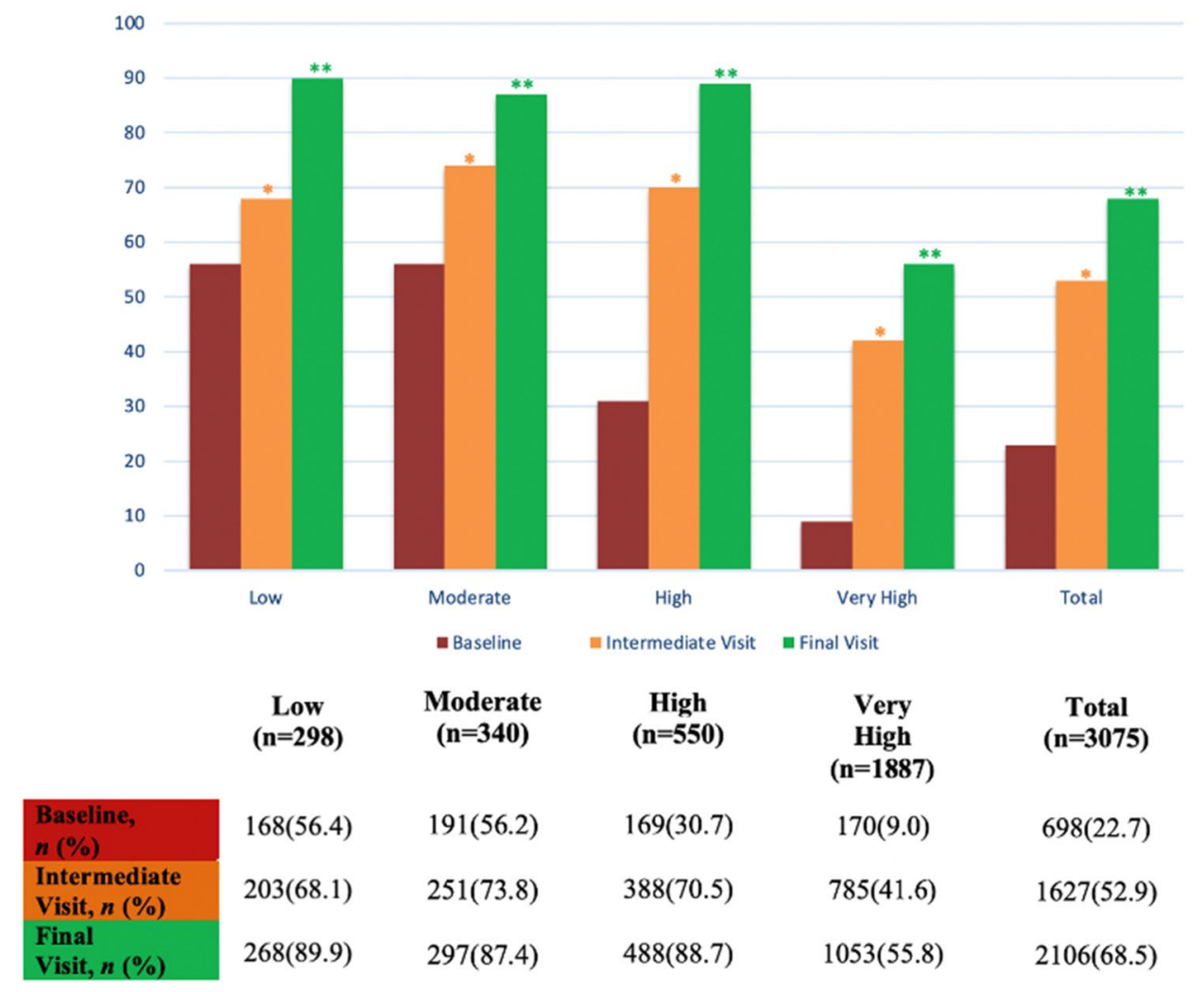

\begin{tabular}{lllll}
\hline & $\begin{array}{l}\text { High } \\
(n=550)\end{array}$ & $\begin{array}{l}\text { Very high } \\
(n=1887)\end{array}$ & $\begin{array}{l}\text { High + very high } \\
(n=2437)\end{array}$ & $\begin{array}{l}\text { Total } \\
(n=3075)\end{array}$ \\
\hline $\begin{array}{l}\text { Major cardiovascular events, } \\
n(\%)\end{array}$ & $5(0.90)$ & $24(1.27)$ & $29(1.24)$ & $29(0.94)$ \\
$\begin{array}{l}\text { Non-fatal myocardial infarction, } n(\%) \\
\text { Non-fatal stroke, } n(\%)\end{array}$ & $3(0.54)$ & $10(0.53)$ & $13(0.53)$ & $13(0.42)$ \\
Other non-fatal ischemic events, $n(\%)$ & 0 & $12(0.64)$ & $15(0.61)$ & $\begin{array}{l}15(0.49) \\
2(0.36)\end{array}$ \\
\hline
\end{tabular}

Table 4 Cardiovascular events in high and very high-risk patients and in study population 
addition of new hypolipidemic drugs) and non-pharmacological interventions. The main therapeutic modifications regarded the add-on of a single tablet statin-ezetimibe combination, which was preferred by physicians in respect to multiple pills as a method to improve adherence [17, 18]. A single tablet statin-ezetimibe combination was taken by $11.8 \%$ of hypercholesterolemic patients at the beginning with an increase to $43.7 \%$ at the end of the follow-up. Likewise, the percentage of patients assuming PCSK-9 inhibitors increased from 1.4 to $12.4 \%$ at the end of the study, mostly prescribed in subjects at very high risk not achieving the required target with the maximum tolerated dose of a statin and ezetimibe (from $2.2 \%$ at the initial visit to $18.4 \%$ at the end of the follow-up). The high and very high-risk patients well tolerated PCSK-9 inhibitors and showed elevated compliance to the therapy, according to the reference randomized trials reporting a marked tolerance and adherence to the treatment with the consequent cardiovascular benefits [19-25]. Non-pharmacological interventions included tailored dietary regimen, planned physical exercise, frequent interactions with the cardiologist and checks of cholesterol levels, patient information and education, e-mail service.

The main findings of our study suggest that the stringent lipid targets recommended by the scientific societies in real-life are achieved by a rather low percentage of patients [26]. A relatively modest number of high or very high-risk patients reaching the LDL-c targets has also been observed in previous studies carried out on large cohorts of outpatients in Italy and other countries [27-29]. However, the promotion and sharing of specific initiatives, emphasizing the effectiveness of cholesterol control and the crucial importance of periodic follow-up, carried out by the referring cardiologists of the pertaining healthcare districts, increase the percentage of patients on target [13,30-32]. In our study the improvement in lipid profile was obtained within a few months thanks to the careful program adopted regarding diet and physical activity, the periodic follow-up, the frequent recurrence of cholesterol dosages, the correct use of risk stratification, the periodic evaluation of the target's achievement and update of the therapies. Finally, these results were also promoted by the opportunity that ARCA cardiologists had to prescribe by themselves the novel, powerful PCSK-9 inhibitors.

ALERT-LDL study suggests that clinicians should assess cardiovascular risk and define precise LDL-c goals at each visit to prescribe the most beneficial therapy early, reducing the time of exposure to high cholesterol levels, which is considered one of the most important components of the cardiovascular risk [5]. Lipid-lowering therapies can reduce the occurrence of cardiovascular events and exert greater health and cost-effectiveness benefits among high-risk people [31]. Thus, public health programs aimed to achieve tailored therapeutic objectives should be aggressively implemented.

\section{Limitations}

The ALERT-LDL registry accurately describes the characteristics of a real-world population of patients consecutively referring to the healthcare territorial districts. The majority of the patients fall into the very high-risk category, limiting the general applicability of the results, but these are the patients who most need to reach the guideline's suggested targets. Moreover, the lack of patients with low to moderate risk might not be due to the characteristics of the target population but related to poor awareness of their increased cardiovascular risk. Therefore, the results of our study suggest that different strategies (f.e. social media) are likely needed to improve patients' awareness and the approach referral to outpatient cardiologists to manage their cardiovascular risk.

\section{Conclusion}

Only one-fifth of dyslipidaemic outpatients referring to the pertaining healthcare districts were on target according to their cardiovascular risk, most of them belonging to the very high-risk group. When included in territorial programs carried on by their cardiologists and specifically aimed at the control of the lipid profile, the percentage of patients on target increased in all risk categories. Thus, the results of this study suggest carefully implementing measures that encourage physicians and patients to achieve the correct lipid profile according to the global cardiovascular risk.

Author contributions GB, MD, UO contributed to the design of the research and wrote the manuscript. GB, GA, VC, FF, BF, FG, AI, SM, MO, ER, GS, AV, GZ, UO collected, analyzed and interpreted the data. All authors read and approved the final manuscript.

Funding Open access funding provided by Università degli Studi di Napoli Federico II within the CRUI-CARE Agreement. The authors received no specific funding for this work.

Data availability The datasets used and/or analyzed during the current study are available from the corresponding author on reasonable request.

\section{Declarations}

Conflict of interest The authors declare that they have no conflict of interest/competing interests.

Consent for publication Not applicable.

Human and animal rights All the enrolled patients signed.

Informed consent informed consent and agreed to participate in a 12-month follow-up program, and the principles outlined in the Declaration of Helsinki were followed. 
Open Access This article is licensed under a Creative Commons Attribution 4.0 International License, which permits use, sharing, adaptation, distribution and reproduction in any medium or format, as long as you give appropriate credit to the original author(s) and the source, provide a link to the Creative Commons licence, and indicate if changes were made. The images or other third party material in this article are included in the article's Creative Commons licence, unless indicated otherwise in a credit line to the material. If material is not included in the article's Creative Commons licence and your intended use is not permitted by statutory regulation or exceeds the permitted use, you will need to obtain permission directly from the copyright holder. To view a copy of this licence, visit http://creativecommons.org/licenses/by/4.0/.

\section{References}

1. Authors/Task Force Members; ESC Committee for Practice Guidelines (CPG); ESC National Cardiac Societies (2019) 2019 ESC/EAS guidelines for the management of dyslipidaemias: Lipid modification to reduce cardiovascular risk. Atherosclerosis 290:140-205

2. Ference BA, Graham I, Tokgozoglu L, Catapano AL (2018) Impact of lipids on cardiovascular health: JACC health promotion series. J Am Coll Cardiol 72:1141-1156

3. Collaboration ERF, Di Angelantonio E, Gao P, Pennells L, Kaptoge S, Caslake $\mathrm{M}$ et al (2012) Lipid-related markers and cardiovascular disease prediction. JAMA 307:2499-2506

4. Ference BA, Ginsberg HN, Graham I, Ray KK, Packard CJ, Bruckert E et al (2017) Low-density lipoproteins cause atherosclerotic cardiovascular disease. 1. Evidence from genetic, epidemiologic, and clinical studies. A consensus statement from the European Atherosclerosis Society consensus panel. Eur Heart J 38(32):2459-2472

5. Domanski MJ, Tian X, Wu CO, Reis JP, Dey AK, Gu Y et al (2020) Time course of LDL cholesterol exposure and cardiovascular disease event risk. J Am Coll Cardiol 76(13):1507-1516

6. Michos ED, McEvoy JW, Blumenthal RS (2019) Lipid Management for the prevention of atherosclerotic cardiovascular disease. N Engl J Med 381(16):1557-1567

7. Baigent C, Keech A, Kearney PM, Blackwell L, Buck G, Pollicino $\mathrm{C}$ et al (2005) Efficacy and safety of cholesterol-lowering treatment: prospective meta-analysis of data from 90,056 participants in 14 randomised trials of statins. Lancet 366(9493):1267-1278

8. Silverman MG, Ference BA, Im K, Wiviott SD, Giugliano RP, Grundy SM et al (2016) Association between lowering LDL-C and cardiovascular risk reduction among different therapeutic interventions: a systematic review and meta-analysis. JAMA 316:1289-1297

9. Mills EJ, Rachlis B, Wu P, Devereaux PJ, Arora P, Perri D (2008) Primary prevention of cardiovascular mortality and events with statin treatments: a network metaanalysis involving more than 65,000 patients. J Am Coll Cardiol 52:1769-1781

10. Collaboration CTT, Baigent C, Blackwell L, Emberson J, Holland LE, Reith C et al (2010) Efficacy and safety of more intensive lowering of LDL cholesterol: a metaanalysis of data from 170,000 participants in 26 randomised trials. Lancet 376:1670-1681

11. Schedlbauer A, Davies P, Fahey T (2010) Interventions to improve adherence to lipid lowering medication. Cochrane Database Syst Rev 3:CD004371

12. Cabana MD, Rand CS, Powe NR, Wu AW, Wilson MH, Abboud PA et al (1999) Why don't physicians follow clinical practice guidelines? A framework for improvement. JAMA 282(15):1458-1465
13. Guarnaccia F, Fimiani B, Zito GB, De Simone A, Stabile G, Bossone $\mathrm{E}$ et al (2014) ALERT-HF: adherence to guidelines in the treatment of patients with chronic heart failure. J Cardiovasc Med (Hagerstown) 15(6):491-497

14. Catapano AL, Graham I, De Backer G, Wiklund O, Chapman MJ, Drexel H (2016) 2016 ESC/EAS guidelines for the management of dyslipidaemias. Eur Heart J 37(39):2999-3058

15. De Luca M, Iacono O, Lucci R, Guardasole V, Bosso G, Cittadini A et al (2020) Atorvastatin-linked rhabdomyolysis caused by the simultaneous intake of amoxicillin clavulanic acid. J Basic Clin Physiol Pharmacol. https://doi.org/10.1515/jbcpp-2020-0108

16. Banach M, Rizzo M, Toth PP, Farnier M, Davidson MH, AlRasadi K et al (2015) Statin intolerance - an attempt at a unified definition. Position paper from an International lipid expert panel. Arch Med Sci 11:1-23

17. Balu S, Simko RJ, Quimbo RM, Cziraky MJ (2009) Impact of fixed-dose and multi-pill combination dyslipidemia therapies on medication adherence and the economic burden of sub-optimal adherence. Curr Med Res Opin 25(11):2765-2775

18. Kamat SA, Bullano MF, Chang CL, Gandhi SK, Cziraky MJ (2011) Adherence to single-pill combination versus multiple-pill combination lipid-modifying therapy among patients with mixed dyslipidemia in a managed care population. Curr Med Res Opin 27(5):961-968

19. Moriarty PM, Thompson PD, Cannon CP, Guyton JR, Bergeron J, Zieve FJ et al (2015) Efficacy and safety of alirocumab vs ezetimibe in statin-intolerant patients, with a statin rechallenge arm: the ODYSSEY ALTERNATIVE randomized trial. J Clin Lipidol 9(6):758-769

20. Iqbal Z, Dhage S, Mohamad JB, Abdel-Razik A, Donn R, Malik R et al (2019) Efficacy and safety of PCSK9 monoclonal antibodies. Expert Opin Drug Saf 18(12):1191-1201

21. Cannon CP, Cariou B, Blom D, McKenney JM, Lorenzato C, Pordy R et al (2015) Efficacy and safety of alirocumab in high cardiovascular risk patients with inadequately controlled hypercholesterolaemia on maximally tolerated doses of statins: the ODYSSEY COMBO II randomized controlled trial. Eur Heart J 36(19):1186-1194

22. Karatasakis A, Danek BA, Karacsonyi J, Rangan BV, Roesle MK, Knickelbine T et al (2017) Effect of PCSK9 inhibitors on clinical outcomes in patients with hypercholesterolemia: a metaanalysis of 35 randomized controlled trials. J Am Heart Assoc 6(12): 0006910

23. Bittner VA, Giugliano RP, Brinton EA, Guyton JR (2018) PCSK9 inhibitors for prevention of atherosclerotic cardiovascular disease. J Clin Lipidol 12(4):835-843

24. Sabatine MS, Giugliano RP, Keech AC, Honarpour N, Wiviott SD, Murphy SA et al (2017) Evolocumab and clinical outcomes in patients with cardiovascular disease. N Engl J Med 376(18):1713-1722

25. Schwartz GG, Steg PG, Szarek M, Bhatt DL, Bittner VA, Diaz R et al (2018) Alirocumab and cardiovascular outcomes after acute coronary syndrome. N Engl J Med 379(22):2097-2107

26. Rosenblit PD (2019) Lowering targeted atherogenic lipoprotein cholesterol goals for patients at "extreme" ASCVD risk. Curr Diab Rep 19(12): 146

27. Presta V, Figliuzzi I, Miceli F, Coluccia R, Fogacci F, Cicero AFG et al (2019) Achievement of low density lipoprotein (LDL) cholesterol targets in primary and secondary prevention: analysis of a large real practice database in Italy. Atherosclerosis 285:40-48

28. Collins R, Reith C, Emberson J, Armitage J, Baigent C, Blackwell $L$ et al (2016) Interpretation of the evidence for the efficacy and safety of statin therapy. Lancet 388(10059):2532-2561

29. De Luca M, Bosso G, Valvano A, Guardasole V, Botta A, Carbone $\mathrm{V}$ et al (2021) Management of patients with chronic heart failure 
and type 2 diabetes mellitus: the SCODIAC-II study. Intern Emerg Med 2021 16(4):895-903

30. Bakke Å, Dalen I, Thue G, Cooper J, Skeie S, Berg TJ et al (2020) Variation in the achievement of HbA1c, blood pressure and LDL cholesterol targets in type 2 diabetes in general practice and characteristics associated with risk factor control. Diabet Med 37(9):1471-1481

31. Kotseva K, Wood D, De Bacquer D, De Backer G, Rydén L, Jennings C et al (2016) EUROASPIRE IV: a European society of cardiology survey on the lifestyle, risk factor and therapeutic management of coronary patients from 24 European countries. Eur J Prev Cardiol 23(6):636-648
32. Bosso G, Valvano A, Guarnaccia F, Fimiani B, Carbone V, Cittadini A et al (2020) Adherence to guidelines in the management of patients with chronic heart failure follow-up: role of periodic echocardiographic examinations. J Cardiovasc Med (Hagerstown) 21(3):216-222

Publisher's Note Springer Nature remains neutral with regard to jurisdictional claims in published maps and institutional affiliations. 